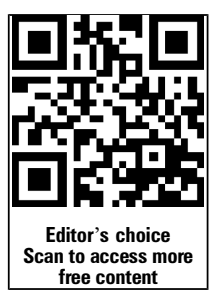

Department of Pharmacy, Northumbria Healthcare NHS Foundation Trust, North Shields, UK

\section{Correspondence to} Dr Wasim Baqir, Department of Pharmacy, Northumbria Healthcare NHS Foundation Trust, North Tyneside Hospital, North Shields NE29 8NH, UK; Wasim.baqir@nhs.net

Received 12 May 2014 Revised 14 August 2014 Accepted 15 August 2014 Published Online First 4 September 2014

\title{
Pharmacist prescribing within a UK NHS hospital trust: nature and extent of prescribing, and prevalence of errors
}

\author{
Wasim Baqir, Olga Crehan, Richard Murray, David Campbell, Richard Copeland
}

Objectives Suitably qualified pharmacists in the UK are able to prescribe all medicines. While doctors' prescribing errors are well documented, there is little information on the rate and nature of pharmacists' prescribing errors. Our aim was to measure the prevalence of prescribing errors by pharmacists. Methods Prescribing by pharmacists, for inpatients admitted to three hospitals in North East England was studied. Part one measured the extent of prescribing by pharmacists as a proportion of all prescribing on a single day. The number of medication orders, reason for prescribing and therapeutic category were collected by the researcher (OC). In part two, pharmacist prescribing was reviewed for safety and accuracy by ward-based clinical pharmacists over 10 days; errors were documented and categorised as per EQUIP study. Results Part 1: Pharmacists prescribed one or more medication orders for 182 (39.8\%) of 457 patients, accounting for $12.9 \%$ (680 from 5274) of all medication orders prescribed on a single census day. Pharmacists prescribed medicines from 12 out of 15 British National Formulary categories (no prescribing of drugs used in malignancy, immunology and anaesthetics). Part 2: 1415 pharmacist-prescribed medication orders were checked by clinical pharmacists, with four errors $(0.3 \%)$ reported. Conclusions This study suggests that prescribing pharmacists can provide a valuable role in safely prescribing for a broad range of inpatients in UK general hospitals.

\section{INTRODUCTION}

Pharmacist supplementary prescribing was introduced in the UK in 2003. ${ }^{1}$ Pharmacist prescribing developed rapidly with the introduction of pharmacist independent prescribing (IP) in 2006, which resulted in pharmacists being able to prescribe, within their competence, except controlled drugs. Since then, successive legislation changes have resulted in pharmacists being able to prescribe across all therapeutic areas, as well as unlicensed medicines and controlled drugs. ${ }^{2}$

The white paper published in 2008, 'Pharmacy in England: Building on Strengths, Delivering the Future', outlined the importance of the pharmacist playing an integral role in the healthcare system and the desire to maximise the skills and roles of the pharmacist in the future. ${ }^{3}$ Recently, the Scottish Government's report highlighted the importance of prescribing pharmacists in the delivery of highquality pharmaceutical care for patients in Scotland. ${ }^{4}$ Generally, pharmacists' prescribing has been positive with many examples of good practice and patient care. ${ }^{1} 5$ Authors have described pharmacist prescribing activity in a number of diverse areas; primary prevention of cardiovascular disease and hypertension, ${ }^{67}$ clinical nutrition ${ }^{8}$ and anticoagulation. ${ }^{9}$ Specifically, hospital pharmacists are prescribing in a number of specific therapeutic areas, including anticoagulation, ${ }^{10}$ antimicrobials, ${ }^{11}$ cardiovascular care, ${ }^{12}$ HIV clinics ${ }^{13}$ as well as having wider roles, for example, pharmaceutical care on surgical wards. ${ }^{14}$ While there is evidence that hospital pharmacists have the self-belief and skills to effectively and safely prescribe in a hospital environment, ${ }^{15}$ there is little published evidence for the extent of prescribing by pharmacists in hospital. While these examples show the diverse range of prescribing undertaken by pharmacists, there are reports of pharmacists who are qualified to prescribe but find they cannot because of lack of defined role or commissioned service, suggesting that further evidence is needed to support the case for prescribing by pharmacists. ${ }^{16}$

A prescribing error has been defined as causing an increase in risk of harm or reduction in the chance of a treatment being timely or effective ${ }^{17}$ and includes transcription errors, failure to communicate essential information and the use of drugs or doses inappropriate for the patient. ${ }^{18}$ Prescribing errors are common in hospitals with a systematic review carried out by Lewis et al reporting a median error rate of $7 \%$ of all inpatient medicines orders. ${ }^{19}$ A UK study of 6605 medicines orders written across three National Health Service (NHS) organisations cited an error rate of $14.7 \%$, with a mean of 0.9 doses being administered before the error was discovered and corrected. ${ }^{20}$ Similar findings were reported from the Scottish PRescribing Outcomes for Trainee Doctors Engaged in Clinical Training (PROTECT) programme, where a review of 4710 patient charts and 47726 medicines showed an average error rate of $7.5 \% .{ }^{21}$ Patients being admitted into hospital were particularly at risk, with many regular medicines being missed or incorrectly prescribed. ${ }^{22}$ Ahmed et al discovered unintentional discrepancies in medicines prescribed in $58 \%$ of patients at the point of admission. ${ }^{23}$

The General Medical Council's EQUIP Study, involving 19 trusts in North-West England, found 11077 errors from 124260 medicines orders $(8.9 \%$ prescribing error rate). ${ }^{22}$ The error rate varied according to prescriber: Foundation Year 1 doctors $8.4 \%$, Foundation Year 2 doctors $10.3 \%$, consultants $5.9 \%$, nurses $6.1 \%$ and pharmacists $0 \% .^{22}$

Pharmacists have been recognised as being key to error identification and reduction. ${ }^{24} 25$ The role of 
hospital pharmacists in identifying and rectifying errors before they reach the patient has been well documented for some time now. ${ }^{26} 27$ Pharmacists corrected or discussed with prescribers the majority of errors reported in a study of doctors' prescribing across three NHS organisations. ${ }^{20}$

It is well recognised that involving pharmacists in the prescribing pathway reduces the risk of an error reaching the patient. ${ }^{28}$ What is less well understood is whether using pharmacists as prescribers also reduces the risk of error. The EQUIP study found no errors by pharmacists, however, the sample size was very low with only 172 medication orders being prescribed by pharmacists. ${ }^{22}$ Our aims were to measure the extent and nature of prescribing by pharmacists as a proportion of all prescribing, and to measure the prevalence of prescribing errors by pharmacists.

\section{METHODS}

The study was undertaken across three North East England district general hospitals belonging to the NHS Foundation Trust where the authors worked. The study comprised of two discrete parts; prevalence of pharmacist prescribing and prevalence of prescribing errors by pharmacists. Both parts of the study were undertaken across all wards (medical, emergency admissions, elderly, surgical, paediatric and the coronary care unit) at the three hospitals. Prescribing pharmacists provide pharmaceutical services to all these wards routinely and this study assessed their usual practice. All prescribing was handwritten on inpatient treatment charts (medicines charts) as there was no electronic system at the hospitals.

\section{Part one}

Part one assessed the prevalence of prescribing by pharmacists. This was done by quantifying the number of medicines orders prescribed by pharmacists as a proportion of all prescribing by all prescribers on a single day. A standardised data collection form was developed and piloted. For the purposes of this study, a medicine order was defined as any medicinal or otherwise (eg, sip-feeds, dressings) that has been prescribed on the inpatient treatment chart (medicines chart) since the patient's admission to hospital.

Data were collected from all wards across the three hospitals by the same researcher between September and October 2012. Over this period, the researcher (OC) visited each ward once and collected information from the inpatient treatment charts (medicines charts) of every patient who was on the ward at that time. Data collection was undertaken on a Friday afternoon and each ward was visited once, with data collection ending when all wards across the three hospitals had been visited. Data collected included the number of medicines taken by the patient, reason for prescribing (existing medicine, new medicine, correction of error, medicine stopped, dose change or whether a medicine was rewritten for clarity) and therapeutic category (British National Formulary, BNF) of the medicine. All medicines, including 'one off' doses, regular medicines and 'when required' medicines were included in the data collection. All prescribing undertaken at any time during the patient's admission was included in the data collection.

Patients were excluded from the study if they were not on the ward at the time of data collection. Data were entered into a spreadsheet and analysed descriptively.

\section{Part two}

Part two was undertaken across all wards across the three hospitals. Ward-based clinical pharmacists, who were not prescribers, were asked to note all prescribing by prescribing pharmacists and clinically assess it for safety and accuracy. This clinical check of prescribing involved clinical pharmacists ensuring that it was correct and safe for the patient, taking into account dose, medicine choice, co-morbidity, interacting medicines, blood results and any other factors that may adversely affect the patient. Additionally, the clinical pharmacists were also asked to highlight prescribing that did not meet the trust's prescribing policy (legible, legal and approved by the formulary committee).

A data collection form was developed and piloted with the clinical pharmacists. The data collection form was developed from the data collected and data collection forms used in the EQUIP study. ${ }^{22}$ Data were collected over 10 days (Monday to Friday) over two consecutive weeks in November 2012. The number of medication orders taken by the patient, reason for prescribing (existing medicine, new medicine, correction of error, medicine stopped, dose change or whether a medicine was rewritten for clarity) and where (clinical speciality) the prescribing occurred was collected.

Prescribing by pharmacists was identified from the signature/ name box on the inpatient treatment chart. Additionally, prescribing pharmacists annotated IP against patients' names on the ward handover documentation in order to highlight to other clinical pharmacists that they had prescribed for those patients. Prescribing pharmacists were fully aware of the study.

An error was identified as any intervention the clinical pharmacist had to make to ensure that the prescribing was clinically correct and legal. The clinical pharmacists were asked to classify the type of error using the 29 error categories used by the authors of the EQUIP study. ${ }^{22}$ These error categories fell into four harm categories: potentially lethal error, serious error, significant error and minor error. Data were entered into a spreadsheet and analysed descriptively.

Advice on ethical approval was sought from the trust's Research Development Unit, which advised that NHS ethical approval was not needed.

\section{RESULTS}

\section{Part one}

A total of 457 patients on 26 wards across three hospitals were included in part one of the study with the pharmacist prescribing for $182(39.8 \%)$ patients. Pharmacists prescribed $12.9 \%$ of all medication orders (680 from 5274 orders). Pharmacists prescribed a wide variety of medicines from 12 out of the 15 BNF therapeutic categories (no prescribing of drugs used in malignancy, immunology and anaesthetics). The majority of prescribing was for central nervous system, cardiovascular and respiratory medicines.

The majority of the prescribing (68.1\%) was done at the point of medicines reconciliation when patients were first admitted and involved prescribing regular medicines that the patient was taking prior to admission (table 1). The remaining prescribing was for new medicines that were started in hospital by the pharmacist $(18.7 \%)$, correcting incorrectly prescribed medicines (7.5\%) and stopping medicines (2.9\%).

\section{Part two}

In part two, 1415 pharmacist-prescribed medication orders were clinically checked by clinical pharmacists over the 10 days, with four errors $(0.3 \%$ error rate) reported. The errors found in this study were: (1) simvastatin $40 \mathrm{mg}$ and amlodipine $10 \mathrm{mg}$ coprescribed (maximum simvastatin dose with calcium channel blocker is now $20 \mathrm{mg}$ ); (2) morphine sulfate $10 \mathrm{mg} / \mathrm{mL}$ solution was prescribed instead of oxycodone $5 \mathrm{mg} / \mathrm{mL}$ solution; (3) 
Table 1 Part one-reason for prescribing by pharmacists

\begin{tabular}{lc} 
Prescribing reason & Medication orders prescribed (\%) \\
\hline Regular medicine not prescribed & $463(68.1)$ \\
New medicine & $127(18.7)$ \\
Incorrectly prescribed medicine & $51(7.5)$ \\
Medicine stopped & $20(2.9)$ \\
Medicine dose change & $10(1.5)$ \\
Rewritten for clarity & $9(1.3)$ \\
Total & $680(100)$ \\
\hline
\end{tabular}

diltiazem coprescribed with simvastatin $40 \mathrm{mg}$; (4) prescribed medicine was not signed by the prescriber.

In part two, data were collected on where the prescribing occurred; $45 \%$ of the prescribing was undertaken in Emergency Care (Medical Admissions), 40\% on surgical and medical base wards, $13 \%$ in surgical preassessment clinics and $2 \%$ was unknown. Table 2 shows a breakdown by reason for prescribing, with regular medicines being the most common reason for prescribing.

\section{DISCUSSION}

Pharmacist prescribing at the three hospital sites in this study is common practice, but little was known of the prevalence of prescribing by pharmacists and the prevalence of errors. This study has demonstrated hospital pharmacists prescribed for $39.8 \%$ of hospital inpatients, prescribing $12.9 \%$ of all medication orders on a single census day.

A mean prescribing error rate of $0.3 \%$ (medication orders) by pharmacist prescribers was demonstrated, which compares favourably with the EQUIP and PROTECT studies. ${ }^{21} 22$ However, it is recognised that this was a pragmatic study, and has a number of limitations. First, there may be bias due to selection of patients; the patients prescribed for were those present on the study weeks, so may not be representative of a standard group of patients, and it is not known whether other prescribers were initiating more complex prescriptions; the level of prescribing errors made by other prescribers was not evaluated, and may not have been as high as the EQUIP study. However, the results from the PROTECT study ${ }^{21}$ suggest that prescribing errors by doctors is a problem that is not just isolated to one part of the UK. It is also not known whether the results are generalisable to other hospitals. Nevertheless, there are few data on the prevalence and error rate of pharmacist prescribing, and this study does provide an indication of these parameters, which warrant further study in other institutions.

Results from parts 1 and 2 of this study show that the main area of prescribing was regular medicines not being prescribed

Table 2 Reason for prescribing by pharmacists

\begin{tabular}{lc}
\hline Reason for prescribing & Medication orders prescribed (\%) \\
\hline Regular medicine not prescribed & $799(56.5)$ \\
New medicine & $184(13.0)$ \\
Incorrectly prescribed medicines & $102(7.2)$ \\
Medicines stopped & $10(0.7)$ \\
Medicine dose change & $27(1.9)$ \\
Rewritten for clarity & $49(3.5)$ \\
Unknown & $244(17.2)$ \\
Total & $1415(100)$ \\
\hline
\end{tabular}

following admission. This is where medicines taken by the patient prior to admission are omitted in error. ${ }^{29}$ This can potentially lead to patient harm from not taking medicines (eg, worsening of symptoms) or receiving interacting medicines, as clinicians are unaware of what the patient was taking. The EQUIP study reported that one in three prescriptions for regular medicines was omitted. ${ }^{22}$ In this aspect, this study demonstrates that prescribing pharmacists perform a key function in improving medicines reconciliation on admission to hospital and thus reducing the occurrence of unintentional omitted doses. This practice is not replicated in all hospitals, where pharmacists are still dependent upon medical staff-often junior-in order to complete full medicines reconciliation following admission.

It is also noteworthy that $13 \%$ of the medication orders prescribed by pharmacists were for new therapy, suggesting that pharmacists are not just using their prescribing rights to correct doctors' errors but are actively managing patients. Further research is needed to identify differences, if any, between the type of medicines prescribed by pharmacists and doctors. However, this study does show that pharmacists are not focusing on a limited formulary of medicines but are prescribing from all but three sections of the BNF. This underlines one of the potential strengths of pharmacists as prescribers, in that their underpinning knowledge gives them a good understanding of a wide range of medicines in many clinical settings. This allows pharmacists to use their prescribing expertise in varied environments such as wards, where they may be expected to prescribe a range of treatments for a range of conditions. This differs from many models of nurse prescribing where the majority of prescribing is specialist, focusing on a narrower range of medicines or a clinicbased model of pharmacist prescribing often seen in primary care. ${ }^{2}$ In both parts of the study, a small proportion of prescribing activity was to stop medicines; stopping medicines (deprescribing) is an essential part of the prescribing pathway, preventing problematic polypharmacy, and can only be performed by prescribers.

The EQUIP study undertook regression analysis to identify errors between the type of prescription and stage of the hospital stay. ${ }^{22}$ Although these data were collected, statistical analysis was not appropriate as only four errors were detected. Of the four errors reported, three were potentially significant according to the EQUIP criteria and one had no impact on patient safety as the prescribing was not signed for. Two errors were for interacting drugs which had the potential to cause patient harm; increased risk of rhabdomyolysis. The final error was the substitution of oxycodone with morphine sulfate for breakthrough pain relief. This could have potentially led to insufficient pain relief. In all three cases, however, the patients came to no actual harm as errors were identified and corrected before they reached the patient.

A further potential limitation of part one of this study was that the data were collected over 2 months with each ward being visited once, rather than all data from all wards being collected at a single point in time. Our approach allowed the same researcher (OC) to undertake the data collection ensuring consistency of data collection. Finally, the prescribing pharmacists knew that the study was being undertaken and, thus, their prescribing accuracy may have potentially been subject to the Hawthorne effect. ${ }^{30}$

This study suggests that prescribing pharmacists can provide a valuable role in safely prescribing for a broad range of inpatients in UK general hospitals, especially at the point of admission where medicines are known to be frequently omitted by admitting medical staff. 
Key messages

\section{What this paper adds}

What is already known on this subject

- Pharmacist prescribers in the UK can prescribe all medicines available to the National Health Service (NHS).

- Prescribing errors (mainly by doctors) remain a problem in the NHS.

- Pharmacists are well recognised for preventing prescribing errors reaching patients.

- There is little research on prevalence of prescribing by pharmacists or prevalence of prescribing errors by pharmacist prescribers.

What this study adds

- Hospital-based pharmacists prescribe for a significant number of patients across a wide therapeutic spectrum.

- Pharmacist-prescribing in UK hospitals appears safe with a low error rate: $0.3 \%$ of medication orders.

- Further larger controlled studies are recommended to validate the results of this small study.

Contributors All authors were involved in the design and implementation of this study. OC was responsible for data collection. RM/WB/OC were responsible for data analysis. WB led the drafting of the manuscript with contribution from all authors, with all authors approving the final version. All authors contributed to rewriting the manuscript following comments from the reviewers.

Competing interests None.

Provenance and peer review Not commissioned; externally peer reviewed.

Data sharing statement All data collected has been presented in the manuscript.

\section{REFERENCES}

1 Tonna AP, Stewart D, West B, et al. Pharmacist prescribing in the UK-a literature review of current practice and research. J Clin Pharm Ther 2007;32:545-56.

2 Baqir W, Miller D, Richardson G. A brief history of pharmacist prescribing in the UK. Eur J Hosp Pharm 2012;19:487-8.

3 Department of Health. Pharmacy in England: building on strengths-delivering the future. 2008. http://wwwdhgovuk/en/Publicationsandstatistics/Publications/ PublicationsPolicyAndGuidance/DH_083815 (accessed 9 Feb 2012).

4 Scottish Government. Prescription for Excellence: A Vision and Action Plan. 2013. http://wwwscotlandgovuk/Resource/0043/00434053pdf (accessed 31 May 2014).

5 Stewart D, MacLure K, Tonna A. Pharmacist prescribing: update on policy, practice and research. Nurse Prescribing 2011;9:458.

6 Bellingham C. Supplementary prescribing in practice. Pharm J 2003;271:144.

7 Smalley L. Supplementary prescribing in action-An example from primary care. Pharm J 2005:274:213-14.

8 Gross Z. Clinical nutrition: pharmacist prescribing of parenteral nutrition. Hosp Pharm 2005;12:17, 19-20.
9 Khoo A, Bajorek BV. Extended roles for pharmacists in warfarin therapy: Identifying opportunities for pharmacist prescribing. J Pharm Pract Res 2006;36:190-3

10 Nguyen NA, Bajorek BV. Pharmacist prescribing in warfarin therapy: Exploring clinical utility in the hospital setting. J Pharm Pract Res 2008;38:35-9.

11 Tonna A. Pharmacist prescribing of antimicrobials in secondary care. Pharm $J$ 2007;279:44-5

12 Donnelly $\mathrm{R}$, Hughes $\mathrm{CM}$, Harper $\mathrm{R}$, et al. A feasibility study of pharmacist independent prescribing in a primary care setting. Diabetic Med 2010;27(2 Suppl 1):37-188.

13 Bellingham $C$. How supplementary prescribing helps in both acute and chronic hospital care. Pharm J 2004:272:640-1.

14 Sassi-Jones K. How non-medical prescribing improved patient care on a surgica ward. Clin Pharm 2010;2:S4-5.

15 Tonna AP, Stewart DC, West B, et al. Exploring pharmacists' perceptions of the feasibility and value of pharmacist prescribing of antimicrobials in secondary care in Scotland. Int J Pharm Pract 2010;18:312-19.

16 Baqir W, Clemerson J, Smith J. Evaluating pharmacist prescribing across the north east of England. Br J Clin Pharm 2010;2:147-9.

17 Avery AA, Barber N, Ghaleb M, et al. Investigating the prevalence and causes of prescribing errors in general practice: the PRACtICe Study. Project Report. General Medical Council, 2012. http://wwwgmc-ukorg/about/research/12996asp (accessed 2 Jun 2014).

18 Dean B. What is a prescribing error? Qual Health Care 2000;9:232-7.

19 Lewis PJ, Dornan T, Taylor D, et al. Prevalence, incidence and nature of prescribing errors in hospital inpatients: a systematic review. Drug Saf 2009;32:379-89.

20 Franklin BD, Reynolds M, Shebl NA, et al. Prescribing errors in hospital inpatients: a three-centre study of their prevalence, types and causes. Postgrad Med $J$ 2011;87:739-45.

21 Ryan C, Ross S, Davey P, et al. Prevalence and causes of prescribing errors: the PRescribing Outcomes for Trainee Doctors Engaged in Clinical Training (PROTECT) study. PLOS ONE 2014;9:e79802.

22 Dornan $\mathrm{T}$, Ashcroft $\mathrm{D}$, Heathfield $\mathrm{H}$, et al. An in depth investigation into causes of prescribing errors by foundation trainees in relation to their medical education. EQUIP study. London: General Medical Counsel, 2009. http://www.gmc-uk.org/ FINAL_Report_prevalence_and_causes_of_prescribing_errors.pdf_28935150.pdf (accessed 13 Apr 2013).

23 Ahmed S, Harding J. Investigating the procedures, drawbacks and implications of seamless primary-secondary care. Quality in Primary Care 2005;13:51-6.

24 Vincent C, Barber N, Dean Franklin B, et al. The contribution of pharmacy to making Britain a safer place. London: Royal Pharmaceutical Society of Great Britain, 2009.

25 Abdel-Qader DH, Harper L, Cantrill JA, et al. Pharmacists' interventions in prescribing errors at hospital discharge: an observational study in the context of an electronic prescribing system in a UK teaching hospital. Drug Saf2010;33:1027-44.

26 Dale A, Copeland R, Barton R. Prescribing errors on medical wards and the impact of clinical pharmacists. Int J Pharm Pract 2003;11:19-24.

27 Batty R, Barber N. Ward pharmacy: a foundation for prescribing audit? Qual Health Care 1992;1:5-9.

28 The Health Foundation. Evidence Scan: Reducing Prescribing Errors, 2012. http:/l www.health.org.uk/public/cms/75/76/313/3337/Reducing\%20prescribing\%20errors \%20-\%20evidence\%20scan.pdf?realName=rMxfeW.pdf (last accessed $12 \mathrm{Apr}$ 2013).

29 Dutton K, Hedger N, Wills S, et al. Prevent medication errors on admission. Clin Governance 2003;8:128-38.

30 Hart CWM. The Hawthorne experiments. In: White KL, ed. Health services research: an anthology. Clinical Governance: An International Journal. Washington: Pan American Health Organization, 1992:29-37. 